\title{
FELICIDAD, OPTIMISMO Y AUTORREALIZACIÓN EN ESTUDIANTES DE UN PROGRAMA DE EDUCACIÓN SUPERIOR PARA ADULTOS
}

\author{
HAPPINESS, OPTIMISM AND SELF-REALIZATION IN STUDENTS FROM A HIGHER EDUCATION \\ PROGRAM FOR ADULTS
}

\author{
Ronald M. Hernández* Y Renzo Felipe Carranza Esteban** \\ *Licenciado en Psicología y Maestrante en Educación. Coordinador de Publicaciones Científicas de la \\ Universidad San Ignacio de Loyola y Editor Adjunto de la Revista Propósitos y Representaciones. \\ Av. La Fontana 750, La Molina, Lima 12, Perú. E-Mail: rhernandezv@usil.edu.pe \\ **Psicólogo y Magister en Educación. Docente en la Facultad de Ciencias de la Salud de la \\ Universidad Peruana Unión (UPeU). E-Mail: renzo.carranza@upeu.edu.pe \\ *Universidad Peruana Unión. Tarapoto - Perú \\ **Universidad San Ignacio de Loyola. Lima - Perú
}

\section{REsumen}

Actualmente se comienzan a estudiar temas novedosos e interesantes que son promovidos por la Psicología Positiva (PP). Esta nueva orientación de la Psicología estudia lo que va bien en la vida, desde el momento en el que el ser humano llega a este mundo hasta que fallece (Seligman \& Csikszentmihalyi, 2000). Seligman después de muchos años de estudiar la depresión, realizó un giro de $180^{\circ}$ para estudiar las emociones positivas, dando lugar a lo que hoy se conoce como Psicología Positiva (Zúñiga, 2010).

Se presenta un estudio que analiza la relación que existe entre felicidad, optimismo y autorrealización, en alumnos de un Programa de Educación Superior para Adultos. Se estudió también la descripción de las variables de estudio, a través de datos sociodemográficos. La muestra estuvo compuesta por 300 sujetos de ambos sexos, de 19 a 59 años de edad. Se administraron la versión revisada y española del Test de Orientación Vital, la Escala de Felicidad de Lima (EFL) y la Escala de Autorrealización APICE de LENI. Las propiedades psicométricas de los instrumentos evidencian su validez y confiabilidad. Entre los princi- pales hallazgos se encontró que existe una correlación directa y altamente significativa entre felicidad y optimismo y felicidad y autorrealización. De igual forma, se observa una distribución normal múltiple de las dimensiones de felicidad, optimismo y autorrealización, las que representan las variables de entrada al análisis de correlación canónica. Se encontró una correlación positiva muy intensa entre el sentido positivo de la vida y una correlación negativa muy intensa con el pesimismo.

Palabras clave: Felicidad; Optimismo; Autorrealización; Universitarios; Estudiantes adultos; Psicología Positiva.

\section{ABSTRACT}

Currently, new and interesting topics are being studied and they are promoted by Positive Psychology. This new psychological approach studies what makes life worth living, analyzes what is going well in life since birth until death (Seligman \& Csikszentmihalyi, 2000). Positive Psychology studies the optimal experience, in which people 
show how they are and do their best in each activity they perform. Thus, a new look at humans, focusing on their positive qualities, welfare, optimism and happiness, this new landscape gives us a new approach to solving the problems of mental health and obtaining optimum quality is provided of life. The results of the Positive Psychology are aimed at contributing to a more complete and balanced scientific understanding of the existence and experience of the human being and transmitting valuable lessons about how to build a happy, healthy, productive and significant life (Park \& Peterson, 2009). Based on the literature review, there are currently few studies addressing topics of Positive Psychology, and of happiness, optimism and selfrealization in adult college students compared to self-concept or anxiety. "Martin Seligman was one of the mentors in relation with this. After he had studied about depression for many years, he started to study positive emotions giving place to what is known today as Positive Psychology" (Zúñiga, 2010, p. 9). A research was done and it analyzes the relationship between happiness, optimism and self-realization in students of a Higher Education Program for Adults. The description of study variables was also studied using sociodemographic data. Samples consisted of 300 adult students of both genders and of age 19 to 59. The reviewed Spanish version of Life Orientation Test, the Happiness Scale of Lima (EFL) and the APICE Self-realization Scale of Leni were applied. The psychometric properties of the instruments show that they are valid and reliable. The validity of the content was obtained from the opinions of experts, and reliability values for the scales. Among the main findings, it was found that there is a direct and highly significant correlation between happiness and optimism, happiness and self-realization and optimism and self-realization, that is, high levels of happiness and optimism are better predictors of self-realization, ie that as college students adults evidencing higher levels of happiness, also presented higher levels of optimism, which allows feel self-realization, so students who are perceived happy and optimistic have less unsolved problems when fighting for their goals, targets or face the difficulties of their environment, performing them in a more successful way. Likewise, there is a multiple normal distribution of happiness, optimism and self-realization dimensions, which re- present input variables to the canonical correlation analysis. A very intense positive correlation was found between positive meaning of life and a very intense negative correlation with pessimism. In conclusion, each stage of the cycle of life in the human being implies getting adapted, setting goals, accepting losses, as well as new challenges; but during the early adulthood stage, the human being experiences and develops physical, cognitive, affective and social changes, which are framed in a single goal, responsibility in the family, work and studies (François, 2001). Thus, people who feel happy, optimistic and self-realized will be more socially affective, more persistent, tenacious in achieving their goals and more competitive and will be more motivated for the effective performance. By focusing on the discussion of this article, the positive relationships they play an important role, are related to social skills which favors the academic development, especially in adults who undertake the challenge of becoming professionals; also it is invited to these results as a basis to highlight the need to expand research with positive variables as its application in academia can help promote the role of a student.

Key words: Happiness; Optimism; Self-realization; College students; Adult students; Positive Psychology.

\section{INTRODUCCIÓN}

En los últimos años se ha denotado el avance investigativo en aquellos recursos psicológicos que favorecen la salud y el bienestar psicológico del ser humano (Remor, Amorós \& Carboles, 2006; Vecina, 2006; Vera-Villarroel, Pávez \& Silva, 2012), siendo evidente la aparición de estudios sobre los recursos, fortalezas y afectos positivos y su relación con las conductas individuales y grupales (Martínez, 2006).

La Psicología Positiva (PP) nace dentro de este aspecto y desde su comienzo en 1998 (Seligman \& Csikszentmihalyi, 2000) el centro de sus aportes se basa en las emociones y 
los rasgos positivos (Gustems \& Sánchez, 2015; Seligman, 2002). Desde esta perspectiva surge como objetivo estudiar a la Psicología Positiva como un complemento de la realidad psicológica y cómo la presencia y promoción de estas características positivas, asociadas a las fortalezas humanas, pueden estar implicadas en la reducción de una variedad de trastornos mentales, funcionando como un amortiguador a este tipo de enfermedades (Seligman \& Csikszentmihalyi, 2000; Vázquez, Hervás \& Ho, 2006; Wood \& Joseph, 2010).

La PP busca centrarse más en la salud mental que en la enfermedad, entendiéndose como salud mental a la experiencia de un bienestar psicológico, sin síntomas que evidencien algún trastorno (Muñoz Valdés, Poblete Tolosa \& Jiménez Figueroa, 2012). Para la PP, el concepto de bienestar sería entendido como la percepción del compromiso con la existencia y cambios en la vida (Ryff, Keyes \& Shmotkin, 2002), donde el crecimiento personal, como parte de la autorrealización, puede considerarse como uno de los principales factores positivos (Díaz et al., 2006). De esta forma el poseer actitudes positivas hacia sí mismo y la aceptación de sus cualidades y el mantenimiento de las mismas aluden al crecimiento y continuo desarrollo de una persona (Chitgian-Urzúa, Urzúa \& Vera-Villarroel, 2013; Ryff, 1989; Ryff et al., 2002). Todos estos indicadores estarían dando cuenta de un funcionamiento psicológico positivo y serían los aspectos centrales para la evaluación del bienestar psicológico y su influencia en sus distintos aspectos.

Cuadra y Florenzano (2003) mencionan que este tipo de bienestar se ha convertido con el paso del tiempo, en una condición dada para el hombre, reflejando así una tendencia social enfocada a nuevas dimensiones que valoran aspectos antes considerados como poco interesantes o de menor importancia, y que actualmente tienen una enorme repercusión positiva en el rendimiento académico, laboral y social. Para Casullo (2002), este bienestar subjetivo es un área de interés científico que se orienta hacia las respuestas emocionales, satisfacción de dominio y juicios globales de satisfacción con la vida, siendo el optimismo y la felicidad herramientas de afrontamiento y defensa cuando el equilibrio se ve amenazado; de esta forma el bienestar correspondería al grado en que una persona juzga favorablemente su vida como un todo.

Para la Psicología Positiva, el optimismo y la felicidad forman un argumento y tema de interés, ya que en muchos casos la orientación que le demos a nuestra vida estará marcada por estas características. La etapa de la adultez temprana es una fase en la cual el ser humano experimenta y desarrolla cambios físicos, cognoscitivos, afectivos y sociales. Es durante estos años que las decisiones que se tomen han de afectar al resto de la vida, con respecto a la salud, la felicidad y el éxito del individuo, donde las principales actividades van dirigidas al logro de objetivos, la responsabilidad en la familia, la vida laboral y académica; en este sentido para la sociedad, estos años son los más importantes de toda la vida. Biswas-Diener y Diener (2006), Chang y Asakawa (2003), González y Restrepo (2009) y Vera-Villarroel, Córdova-Rubio y Celis-Atenas (2009) mencionan que depende de su percepción del contexto cultural y económico que lo rodea para que una persona se sienta satisfecha y feliz. De esta manera, factores como la educación, el ambiente cultural y los antecedentes familiares pueden impactar en la fuerza e importancia sobre una necesidad, generando así un estado de bienestar y satisfacción con la vida (Alarcón, 2000; Cuadra \& Florenzano, 2003).

Es indudable entonces, que la felicidad y el optimismo como variables psicológicas brindan una orientación optimista a la vida de una persona, mientras que la autorrealización consiste en el desarrollo y la expresión adecuada de las capacidades, de modo que la persona pueda vivir una vida satisfactoria y de provecho para sí misma y para otras personas. Implican la búsqueda del cumplimiento de las metas que conforman el proyecto vital de su existencia (Naranjo, 2007). Sin embargo, dentro del contexto actual y en el cual se enmarca el estudio que se informa, el desempeño académico de un adulto se ha visto influenciado por la competitividad y la exigen- 
cia laboral, que han generado una presión social progresiva con el paso de los años. Actualmente, la necesidad de conservar un trabajo, de construir una imagen competente ante los jefes y de la satisfacción personal se han convertido en factores potencializadores de estrés, depresión, ansiedad, irritabilidad y de diversos trastornos mentales (Cruz et al., 2010).

Ciertamente, sería interesante conocer en qué medida estas variables contribuyen directa e indirectamente al logro académico de un estudiante. Así en la mayoría de las investigaciones se ha constatado una implicancia directa y de mayor relevancia, entre el bienestar psicológico y las competencias necesarias para el logro de los objetivos propuestos. Del mismo modo, si bien la preeminencia del funcionamiento social es innegable en la repercusión académica, algunos antecedentes permiten pensar que las emociones positivas podrían tener un influjo similarmente potente en el desarrollo de ciertas competencias estudiantiles y en las habilidades sociales (Oros \& Fontana, 2015).

Se han destacado estudios como el de Grimaldo (2004), que indican que aquellos alumnos que presentan niveles promedio de optimismo, tienen una tendencia a esperar resultados positivos o favorables en sus vidas y expectativas generalizadas de resultados positivos. Salgado (2009) estudió en una muestra de estudiantes de educación secundaria, la relación entre resiliencia, felicidad y optimismo, encontrando una relación directa y altamente significativa entre las tres variables, deduciendo que una persona feliz refleja altos niveles de pensamientos positivos que revelan su optimismo, así como habilidades para salir adelante a pesar de las diversas dificultades que pueda vivir. Torres, Moyano y Páez (2014) exponen que estudiantes universitarios con altos niveles de felicidad, reportan un desempeño, sociabilidad y rendimiento óptimo en el ámbito académico. Arequipeño y Lastra (2016) explican que el $89.1 \%$ de una población de 213 estudiantes reporta niveles altos de felicidad con adecuados mecanismos de afrontamiento y mejor disposición para lograr sus objetivos de vida. Mus- taca, Kamenetzky y Vera (2010) evaluaron las diferencias entre sujetos optimistas y no optimistas, en variables negativas (Depresión y Ansiedad) y en variables positivas (Satisfacción con la vida, Felicidad y Autoestima) y concluyen que es necesario explorar la utilización del optimismo y otras variables positivas estudiadas en los últimos años, como herramientas para la psicoterapia, intervenciones clínicas y prevención de la salud. En esta misma línea la felicidad sería la variable que se asocia con el optimismo, aumento de alegría, satisfacción, nivel de energía, mejora del sistema inmunológico, dedicación al trabajo y salud mental (Lyubomirsky, 2008; Moyano, Flores \& Soromaa, 2011, Vera-Villarroel, Celis-Atenas, Pavez, Lillo, Bello, Díaz \& López, 2012). Para Peterson (2000), dicha interpretación involucra niveles cognitivos, emocionales y motivadores que estimulan la autorrealización, estimulando la perseverancia, resistencia mental y física y la disposición hacia el éxito. Asimismo, manifiesta que el ser humano necesita sentirse optimista en algunas materias de la vida para surgir y cumplir sus metas. En el caso de los adultos mayores, Marrero, Carballeira y González (2014) indican que las personas que se caracterizan por puntuar alto en optimismo también muestran una mayor puntuación en todos los indicadores de bienestar, frente a las que se sitúan en posiciones intermedias o presentan bajo optimismo. Castaño y Garín (2012) manifiestan que gracias al optimismo, los estudiantes universitarios adultos reportan una resistencia a la sobrecarga estresora, considerando que tienen una familia que mantener, trabajos con los cuales cumplir, dejando de estudiar hace mucho tiempo y con muy poca disponibilidad de tiempo, pero que a pesar de todo ello, la gran mayoría de ellos tiene una buena predisposición e interés en continuar.

A pesar de toda la evidencia, existe aún poca información sobre los mecanismos y las variables relacionadas con los procesos de felicidad, optimismo y afectos positivos, incluyendo tanto los mecanismos psicológicos como los fisiológicos (Davidson, Mostofsky $\&$ Whang, 2010). De acuerdo a esta situación, parece relevante analizar e identificar aque- 
llos factores positivos que en conjunto conforman los factores protectores para el desempeño académico de un estudiante. Igualmente, este tipo de estudio podría ayudar a clarificar y comprender si existen diferencias entre la felicidad y el optimismo como factores significativos en la autorrealización de un estudiante universitario.

Los objetivos propuestos para la realización del estudio que se informa fueron los siguientes: (1) determinar la relación que existe entre felicidad, optimismo y autorrealización en estudiantes universitarios de un Programa de Educación Superior para Adultos y (2) analizar las variables a estudiar según datos sociodemográficos de los estudiantes de dicho programa. Se espera que se determine la existencia de una relación significativa entre la felicidad, optimismo y autorrealización en estudiantes universitarios.

\section{MÉTOdo}

\section{PARTICIPANTES}

La selección de los participantes se realizó con un muestreo absoluto y estuvo compuesta por 300 estudiantes (144 varones y $156 \mathrm{mu}-$ jeres) que se encontraban matriculados en el Programa Superior para Adultos de la Universidad Privada de Tarapoto (Perú), con edades comprendidas entre 19 y 59 años.

Se aprecia en la Tabla 1 que el $57.30 \%$ de los participantes eran mujeres y el $42.70 \%$ hombres, cuya edad promedio oscilaba entre los 19 y 34 años (62\%). Por otro lado, el 55\% de los sujetos eran estudiantes de la Escuela Profesional de Administración y Negocios Internacionales y el $45 \%$ de Contabilidad. El $57 \%$ eran solteros y el $35.70 \%$, casados. En cuanto al lugar de procedencia, el $82.30 \%$ provenía de la selva.

\section{INSTRUMENTOS}

1.- Para la evaluación de felicidad se aplicó la Escala de Felicidad de Alarcón (2006) que contiene 27 ítems distribuido en cuatro di- mensiones (Sentido positivo de la vida, Satisfacción con la vida, Realización personal y Alegría de vivir). El rango de respuestas oscila entre 1 (desacuerdo) y 5 (totalmente de acuerdo). Sus propiedades psicométricas indican que el instrumento es válido y confiable $(r=.511, p<.001 ; \alpha=.916)$.

2.- Para evaluar el optimismo se administró el Test de Orientación Vital revisado (Otero-López, Luengo, Romero, Gómez \& Castro, 1998). Está distribuido en dos dimensiones (pesimismo y optimismo) y conformado por 10 ítems. El rango de respuestas oscila entre 1 (desacuerdo) y 5 (totalmente de acuerdo). Los niveles de validez y consistencia interna indican que el instrumento es válido y confiable $(r=.458, p<.001 ; \alpha=.756)$.

3.- También se administró la Escala APICE de LENI (Burga, Bolaños, Gaspar \& Jara, 2014). Este instrumento mide autorrealización. Contiene cuatro dimensiones (Conciencia, Honestidad, Libertad y Confianza) y 35 ítems. El rango de respuestas oscila entre 1 (nunca) y 5 (siempre). Las propiedades psicométricas indican su validez y confiabilidad $(r=.698, p<.001 ; \alpha=.898)$.

\section{Procedimiento}

La administración de los instrumentos fue colectiva y estuvo supervisada por un investigador principal.

La participación de los estudiantes fue voluntaria y anónima. Se entregó a cada participante un consentimiento informado, para su respectiva lectura y firma, respetando así los principios éticos. Se explicitaron los objetivos de la investigación aclarando que los datos serían analizados sin identificación individual y que su tratamiento sería confidencial. La evaluación se realizó en lugares, fechas y horarios acordados entre el equipo de investigadores y la dirección del programa, respetando las condiciones físicas básicas para concretar esta tarea (escritorio, sillas, adecuada iluminación, niveles de ruidos moderados y privacidad). 


\section{ANÁLISIS DE DATOS}

En primer lugar se realizó un análisis descriptivo de todas las variables, según datos sociodemográficos. Así como también se analizaron las correlaciones de Pearson entre las variables objeto de estudio. El análisis de datos se realizó con el programa estadístico SPSS ${ }^{\circledR}$ versión 20 , estableciéndose un nivel de significancia de .05 (5\%).

\section{Resultados}

El $46.3 \%$ de las mujeres presenta un nivel promedio de felicidad, asimismo, en las frecuencias absolutas se observa que la muestra presenta en mayor proporción un nivel promedio de felicidad, seguido de felicidad baja y alta, específicamente un $84 \%$ del total se encuentra en el nivel de felicidad alta (ver Tabla 2).

En la Tabla 3 se presentan las frecuencias absolutas de optimismo, el $63.7 \%$ del total de la muestra tiene un nivel promedio de optimismo seguido de optimismo alto y bajo. Con respecto a la autorrealización, las frecuencias absolutas muestran en mayor proporción un nivel alto, seguido de autorrealización promedio y bajo, específicamente el $95 \%$ se encuentra en el nivel de autorrealización alto (ver Tabla 4).

La Tabla 5 muestra los niveles globales de las variables de estudio, encontrándose que el $63.6 \%$ de los estudiantes presenta un nivel promedio en la Escala de Optimismo. Un $83.7 \%$ presenta un nivel promedio en la Escala de Felicidad y el nivel promedio para la Escala de Autorrealización fue igual a 4.1\%.

En el análisis de correlación entre felicidad, optimismo y autorrealización ( $r$ de Pearson) se encontró que existe una correlación directa y altamente significativa $(r=.470 ; p<.00)$ entre felicidad, optimismo y felicidad y autorrealización significativa $(r=.270 ; p<.00)$, lo que indica que cuanto más feliz se sienta la persona, más se incrementan los niveles de optimismo y autorrealización. De manera similar ocurre entre optimismo y autorrealización $(r=.128 ; p<.036)$ lo que indica que a mayor optimismo mayores niveles de autorrealización.

La Tabla 6 muestra la presencia de correlaciones directas entre las dimensiones de felicidad, optimismo y autorrealización, como así también una correlación inversa entre el sentido positivo de la vida y el pesimismo.

Para realizar los análisis correlacionales, se aplicó la prueba de esfericidad de BartlettLawley y se observó una evidencia estadística altamente significativa $(p<.01)$ de una distribución normal múltiple de las dimensiones de felicidad, optimismo y autorrealización, las que representan las variables de entrada al análisis de correlación canónica.

En la Tabla 7 se muestra la matriz estructural construida en base a las correlaciones entre las variables de entrada y las canónicas. La correlación más negativa entre la primera variable canónica F1 con el optimismo (-.95) y la satisfacción con la vida (-.93); de forma similar con la alegría de vivir (-.79), realización personal (-.77), con menor intensidad con la libertad (-.53), conciencia (-.43), confianza (-.31), honestidad (-.27), dejándose notar un factor común referido a la Insatisfacción con la vida el cual explicaría un $69.52 \%$ de la variabilidad en este primer grupo de variables de entrada. Así también se encontró una correlación positiva muy intensa con el Sentido positivo de la vida (.96) y una correlación negativa muy intensa (-.83) con el Pesimismo, notándose un factor común referido a la Expectativa positiva de la vida, el cual explica un $24.28 \%$ de la variabilidad de este segundo grupo de variables de entrada, acumulando un total de $93.8 \%$ de variancia explicada por ambas variables canónicas.

En la Figura 1, las dimensiones Optimismo y Satisfacción con la vida se ubican en el lado negativo del eje $\mathrm{X}(\mathrm{F} 1)$, dando a entender que hacia el lado positivo crece lo opuesto a dichas variables, de tal modo que la primera variable canónica estaría referida a la Insatisfacción con la vida, mientras que la dimensión Sentido positivo de la vida se muestra hacia el lado positivo y el pesimismo hacia el lado negativo del eje Y (F2) haciendo referencia a la Expectativa positiva de la vida. 


\section{Discusión}

Las correlaciones muestran que existe una relación directa y significativa entre felicidad y optimismo sobre la autorrealización en los estudiantes universitarios adultos, es decir que en la medida que evidencien mayores niveles de felicidad, también presentarán mayores niveles de optimismo, lo que permite que se sientan realizados.

En la actualidad se estudian temas novedosos e interesantes que son promovidos por la Psicología Positiva. Esta nueva orientación de la Psicología analiza lo que va bien en la vida, desde el momento en el que el ser humano llega a este mundo hasta que fallece (Seligman \& Csikszentmihalyi, 2000). La PP estudia la experiencia óptima, donde las personas se muestran como son y dan lo mejor de sí mismas en cada actividad que realizan. Los resultados de los estudios de la Psicología Positiva tienen como propósito contribuir a una comprensión científica más completa y equilibrada de la existencia y experiencia del ser humano y transmitir enseñanzas valiosas acerca de cómo edificar una vida feliz, saludable, productiva y significativa (Park \& Peterson, 2009).

En este sentido, tras la revisión de la literatura se aprecia que en la actualidad existen todavía pocos estudios que aborden temas de la Psicología Positiva (Seligman, 2003) y con respecto a felicidad, optimismo y autorrealización en estudiantes universitarios adultos son escasos o no existen en comparación con otros constructos (autoestima, depresión, etc.). La importancia del estudio radica en la evidencia empírica que aporta a la PP, entendiendo que la felicidad y el optimismo son importantes para la autorrealización sobre todo en personas adultas de diversa condición, de diversos lugares del Perú y que se encuentran trabajando y deben organizar su tiempo y espacio para alcanzar una carrera profesional, que forma parte de su proyecto de vida, y si no se sienten felices u optimistas, es probable que no logren el éxito.

El objetivo del estudio realizado fue determinar la relación que existe entre felicidad, optimismo y autorrealización en estudiantes universitarios de un Programa de Educación
Superior para Adultos, y se encontró que la felicidad y el optimismo guardan una relación altamente significativa sobre la autorrealización. Un aspecto que se debe tener presente es que un mayor nivel de felicidad permite a la persona ser más optimista, y probablemente esto influya en los momentos de autorrealización. En este sentido, los estudiantes universitarios adultos que se perciban felices y optimistas tendrán menos problemas irresueltos y al momento de luchar por sus objetivos, metas o encarar las dificultades de su entorno lo harán de una forma más exitosa.

En el análisis de correlación se encontró que la felicidad se relaciona significativamente con el optimismo. Esto coincide con los estudios de Grimaldo (2004) y Salgado (2009), quienes sostienen que una persona feliz reflejará altos niveles de optimismo, con una tendencia a mostrar expectativas y resultados positivos. Por lo tanto el optimismo sería una emoción positiva que promueve el bienestar psicosomático lo que ayuda a mantener la homeostasis en circunstancias de estrés y ansiedad (Vera-Villarroel, Córdova-Rubio \& Celis-Atenas, 2009).

En cuanto al optimismo, se encontró que se relaciona significativamente con la autorrealización de los estudiantes universitarios. Los resultados obtenidos se asemejan a los encontrados por Pavez, Mena y Vera-Villarroel (2012), quienes indican que el optimismo sería un factor importante para la autorrealización. De esta forma, una de las características de estas variables se relaciona con el cumplimiento de objetivos trazados en el proyecto de vida (Naranjo, 2007) y su implicancia para la disposición para el éxito (Peterson, 2000).

Con respecto a las diferencias por género, las participantes mujeres obtuvieron puntajes más altos en felicidad, y esto coincide con los estudios de Marrero, Carballeira y González (2014) y Torres y colaboradores (2014), quienes encontraron que ellas puntúan significativamente más que los hombres. Esto parece ser una diferencia importante a considerar ya que se puede empezar a comprender que las mujeres perciben mayores niveles de felicidad, posiblemente debido a que experimentan emociones positivas con mayor intensidad. 
Así también Hartog y Oosterbeek (1996) indican que las mujeres son claramente más felices que los varones. En este sentido, se ha encontrado en diversas investigaciones que la mujeres son más sensibles a las emociones y que responden a estas con empatía y felicidad (Mestre, Samper, Frías \& Tur, 2009). De manera similar ocurre con el optimismo con respecto a las diferencias por género, evidenciándose que las participantes obtuvieron puntajes más altos; estos resultados coinciden con el estudio de Marrero y colaboradores (2014) en el que las mujeres presentaron puntajes más altos de optimismo en comparación con el grupo de varones.

Con respecto a las limitaciones, la investigación destaca que sólo se consideraron estudiantes adultos de Administración y Contabilidad, por lo que resultaría importante ampliar la muestra a otras carreras profesionales, ya que el perfil del ingresante y las exigencias académicas en cada carrera son distintas, por lo tanto los resultados no deben extrapolarse a otro tipo de poblaciones, como por ejemplo, clínicas, pacientes con algún tipo de trastorno mental u organizacionales, trabajadores empleadores y / o empleados.

A pesar de estas limitaciones, existe concordancia frente a los aspectos teóricos, sugiriendo la validez de los resultados obtenidos. Dado que es un estudio correlacional y descriptivo, no se pueden sacar conclusiones causales, entonces deberían realizarse estudios longitudinales que permitan examinar los beneficios de la felicidad, optimismo y autorrealización en estudiantes universitarios.

La Psicología Positiva dentro de sus características principales busca reconsiderar las potencialidades humanas, como factor protector ante momentos de crisis, considerando que las crisis son inevitables y necesarias para el crecimiento y madurez de la persona (Cuadra \& Florenzano, 2003). En este sentido, se sugiere trabajar con otras variables tales como motivación, esperanza, logro, metas, valores, entre otras, de manera que se dé un mayor entendimiento a las variables estudiadas en esta investigación, cuyo objetivo posterior es desarrollar programas de intervención que puedan ayudar a las personas a aprender a ser felices. 
TABLA 1

CARACTERÍSTICAS SOCIODEMOGRÁFICAS DE LOS ESTUDIANTES

\begin{tabular}{|c|c|c|c|}
\hline & Variables & $n$ & $\%$ \\
\hline Género & $\begin{array}{l}\text { Masculino } \\
\text { Femenino }\end{array}$ & $\begin{array}{l}128 \\
172\end{array}$ & $\begin{array}{l}42.70 \\
57.30\end{array}$ \\
\hline Edad & $\begin{array}{l}\text { 19-31 años } \\
\text { 32-45 años } \\
46-59 \text { años }\end{array}$ & $\begin{array}{l}186 \\
83 \\
31\end{array}$ & $\begin{array}{l}62.00 \\
27.70 \\
10.30\end{array}$ \\
\hline $\begin{array}{l}\text { Escuela } \\
\text { profesional }\end{array}$ & $\begin{array}{l}\text { Contabilidad y Finanzas } \\
\text { Administración y Negocios Internacionales }\end{array}$ & $\begin{array}{l}135 \\
165\end{array}$ & $\begin{array}{l}45.00 \\
55.00\end{array}$ \\
\hline Estado civil & $\begin{array}{l}\text { Soltero } \\
\text { Casado } \\
\text { Otro }\end{array}$ & $\begin{array}{c}171 \\
107 \\
22\end{array}$ & $\begin{array}{r}57.00 \\
35.70 \\
7.30\end{array}$ \\
\hline $\begin{array}{l}\text { Lugar de } \\
\text { procedencia }\end{array}$ & $\begin{array}{l}\text { Costa } \\
\text { Sierra } \\
\text { Selva } \\
\text { Extranjero }\end{array}$ & $\begin{array}{c}35 \\
16 \\
247 \\
2\end{array}$ & $\begin{array}{r}11.70 \\
5.30 \\
82.30 \\
.70\end{array}$ \\
\hline
\end{tabular}


TABLA 2

NiVELES DE FELICIDAD SEGÚN DATOS SOCIODEMOGRÁFICOS

\begin{tabular}{|c|c|c|c|c|c|c|c|}
\hline & \multirow{2}{*}{ Variables } & \multicolumn{2}{|c|}{ Bajo } & \multicolumn{2}{|c|}{ Promedio } & \multicolumn{2}{|c|}{ Alto } \\
\hline & & $n$ & $\%$ & $n$ & $\%$ & $n$ & $\%$ \\
\hline \multirow[t]{2}{*}{ Género } & Masculino & 15 & 5.00 & 113 & 37.70 & 0 & .00 \\
\hline & Femenino & 32 & 10.70 & 139 & 46.30 & 1 & .30 \\
\hline \multirow[t]{3}{*}{ Edad } & 19-31 años & 29 & 9.70 & 156 & 52.00 & 1 & .30 \\
\hline & $32-45$ años & 13 & 4.30 & 70 & 23.30 & 0 & .00 \\
\hline & 46-59 años & 5 & 1.70 & 26 & 8.70 & 0 & .00 \\
\hline \multirow{3}{*}{$\begin{array}{l}\text { Escuela } \\
\text { profesional }\end{array}$} & Contabilidad y Finanzas & 24 & 8.00 & 110 & 36.70 & 1 & .30 \\
\hline & Administración y & & & & & & \\
\hline & Negocios Internacionales & 23 & 7.70 & 142 & 47.30 & 0 & .00 \\
\hline \multirow[t]{3}{*}{ Estado civil } & Soltero & 21 & 7.00 & 149 & 49.70 & 1 & .30 \\
\hline & Casado & 24 & 8.00 & 83 & 27.70 & 0 & .00 \\
\hline & Otro & 2 & .70 & 20 & 6.70 & 0 & .00 \\
\hline \multirow{5}{*}{$\begin{array}{l}\text { Lugar de } \\
\text { procedencia }\end{array}$} & Costa & 8 & 2.70 & 27 & 9.00 & 0 & .00 \\
\hline & Sierra & 1 & .30 & 14 & 4.70 & 1 & .30 \\
\hline & Selva & 36 & 12.00 & 211 & 70.30 & 0 & .00 \\
\hline & Extranjero & 2 & .70 & 0 & 0.00 & 0 & .00 \\
\hline & Total & 47 & 15.70 & 252 & 84.00 & 1 & .30 \\
\hline
\end{tabular}


TABLA 3

NivelES DE OPTIMISMO SEGÚN DATOS SOCIODEMOGRÁFICOS

\begin{tabular}{|c|c|c|c|c|c|c|c|}
\hline & \multirow{2}{*}{ Variables } & \multicolumn{2}{|c|}{ Bajo } & \multicolumn{2}{|c|}{ Promedio } & \multicolumn{2}{|c|}{ Alto } \\
\hline & & $n$ & $\%$ & $n$ & $\%$ & $n$ & $\%$ \\
\hline \multirow[t]{2}{*}{ Género } & Masculino & 8 & 2.70 & 83 & 27.70 & 37 & 12.30 \\
\hline & Femenino & 14 & 4.70 & 108 & 36.00 & 50 & 16.70 \\
\hline \multirow[t]{3}{*}{ Edad } & 19-31 años & 11 & 3.70 & 122 & 40.70 & 53 & 17.70 \\
\hline & 32-45 años & 9 & 3.00 & 50 & 16.70 & 24 & 8.00 \\
\hline & 46-59 años & 2 & .70 & 19 & 6.30 & 10 & 3.30 \\
\hline \multirow{3}{*}{$\begin{array}{l}\text { Escuela } \\
\text { profesional }\end{array}$} & Contabilidad y Finanzas & 8 & 2.70 & 87 & 29.00 & 40 & 13.30 \\
\hline & Administración y & & & & & & \\
\hline & Negocios Internacionales & 14 & 4.70 & 104 & 34.70 & 47 & 15.70 \\
\hline \multirow[t]{3}{*}{ Estado civil } & Soltero & 15 & 5.00 & 114 & 38.00 & 42 & 14.00 \\
\hline & Casado & 6 & 2.00 & 62 & 20.70 & 39 & 13.00 \\
\hline & Otro & 1 & .30 & 15 & 5.00 & 6 & 2.00 \\
\hline \multirow{5}{*}{$\begin{array}{l}\text { Lugar de } \\
\text { procedencia }\end{array}$} & Costa & 2 & .70 & 27 & 9.00 & 6 & 2.00 \\
\hline & Sierra & 0 & .00 & 8 & 2.70 & 8 & 2.70 \\
\hline & Selva & 20 & 6.70 & 154 & 51.30 & 73 & 24.30 \\
\hline & Extranjero & 0 & .00 & 2 & .70 & 0 & .00 \\
\hline & Total & 22 & 7.30 & 191 & 63.70 & 87 & 29.00 \\
\hline
\end{tabular}


TABLA 4

NIVELES DE AUTORREALIZACIÓN SEGÚN DATOS SOCIODEMOGRÁFICOS

\begin{tabular}{|c|c|c|c|c|c|c|c|}
\hline & \multirow[b]{2}{*}{ Variables } & \multicolumn{2}{|c|}{ Bajo } & \multicolumn{2}{|c|}{ Promedio } & \multicolumn{2}{|c|}{ Alto } \\
\hline & & $n$ & $\%$ & $n$ & $\%$ & $n$ & $\%$ \\
\hline \multirow[t]{2}{*}{ Género } & Masculino & 3 & 1.00 & 1 & .30 & 124 & 41.30 \\
\hline & Femenino & 0 & 0.00 & 11 & 3.70 & 161 & 53.70 \\
\hline \multirow[t]{3}{*}{ Edad } & 19-31 años & 0 & 0.00 & 10 & 3.30 & 176 & 58.70 \\
\hline & $32-45$ años & 3 & 1.00 & 2 & .70 & 78 & 26.00 \\
\hline & 46-59 años & 0 & 0.00 & 0 & .00 & 31 & 10.30 \\
\hline \multirow[t]{2}{*}{$\begin{array}{l}\text { Escuela } \\
\text { profesional }\end{array}$} & $\begin{array}{l}\text { Contabilidad y Finanzas } \\
\text { Administración y }\end{array}$ & 3 & 1.00 & 4 & 1.30 & 128 & 42.70 \\
\hline & Negocios Internacionales & 0 & 0.00 & 8 & 2.70 & 157 & 52.30 \\
\hline \multirow[t]{3}{*}{ Estado civil } & Soltero & 0 & 0.00 & 2 & .70 & 169 & 56.30 \\
\hline & Casado & 3 & 1.00 & 10 & 3.30 & 94 & 31.30 \\
\hline & Otro & 0 & 0.00 & 0 & .00 & 22 & 7.30 \\
\hline \multirow{5}{*}{$\begin{array}{l}\text { Lugar de } \\
\text { procedencia }\end{array}$} & Costa & 0 & 0.00 & 0 & .00 & 35 & 11.70 \\
\hline & Sierra & 0 & 0.00 & 1 & .30 & 15 & 5.00 \\
\hline & Selva & 3 & 1.00 & 11 & 3.70 & 233 & 77.70 \\
\hline & Extranjero & 0 & 0.00 & 0 & .00 & 2 & .70 \\
\hline & Total & 3 & 1.00 & 12 & 4.00 & 285 & 95.00 \\
\hline
\end{tabular}

TABLA 5

NIVELES GLOBALES DE VARIABLES DE ESTUDIO

\begin{tabular}{|l|c|c|c|}
\hline \multicolumn{1}{|c|}{ Niveles / Variables } & $\begin{array}{c}\text { Optimismo } \\
\%\end{array}$ & $\begin{array}{c}\text { Felicidad } \\
\%\end{array}$ & $\begin{array}{c}\text { Autorrealización } \\
\%\end{array}$ \\
\hline Bajo & 7.3 & 15.7 & 1.0 \\
Promedio & 63.7 & 84.0 & 4.0 \\
Alto & 29.0 & 0.3 & 95.0 \\
Total & 100 & 100 & 100 \\
\hline
\end{tabular}




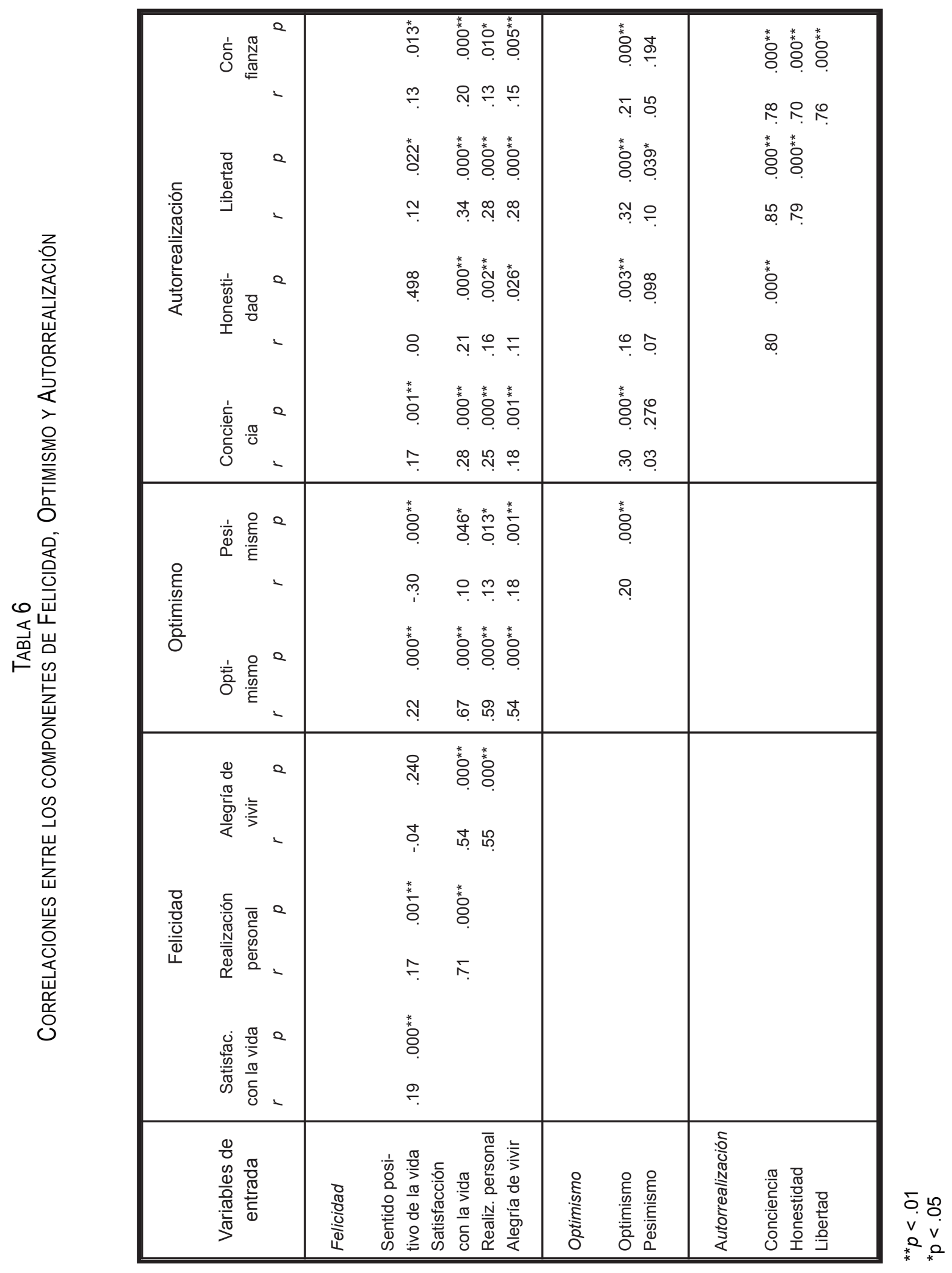




\section{TABLA 7 \\ MATRIZ ESTRUCTURAL}

CORRELACIONES ENTRE VARIABLES DE ENTRADA Y VARIABLES CANÓNICAS

\begin{tabular}{|c|c|c|}
\hline Variables de entrada & $\begin{array}{c}\text { F1 Varia } \\
\text { Insatisfacción con la vida }\end{array}$ & $\begin{array}{l}\text { nónicas } \\
\qquad \text { F2 } \\
\text { Expectativa positiva de la vida }\end{array}$ \\
\hline $\begin{array}{l}\text { Felicidad } \\
\text { Sentido positivo de la vida } \\
\text { Satisfacción con la vida } \\
\text { Realización personal } \\
\text { Alegría de vivir }\end{array}$ & $\begin{array}{l}-.26 \\
-.93 \\
-.77 \\
-.79\end{array}$ & $\begin{array}{l}.96 \\
-.06 \\
-.11 \\
-.23\end{array}$ \\
\hline $\begin{array}{l}\text { Optimismo } \\
\text { Optimismo } \\
\text { Pesimismo }\end{array}$ & $\begin{array}{l}-.95 \\
-.15\end{array}$ & $\begin{array}{l}-.07 \\
-.83\end{array}$ \\
\hline $\begin{array}{l}\text { Autorrealización } \\
\text { Conciencia } \\
\text { Honestidad } \\
\text { Libertad } \\
\text { Confianza }\end{array}$ & $\begin{array}{l}-.43 \\
-.27 \\
-.53 \\
-.31\end{array}$ & $\begin{array}{r}.23 \\
-.14 \\
.05 \\
.20\end{array}$ \\
\hline $\begin{array}{l}\% \text { Variancia } \\
\% \text { Variancia acumulada }\end{array}$ & 69.52 & 24.28 \\
\hline
\end{tabular}


FIGURA 1

Gráfico de Correlaciones de las Variables de entrada y Variables Canónicas

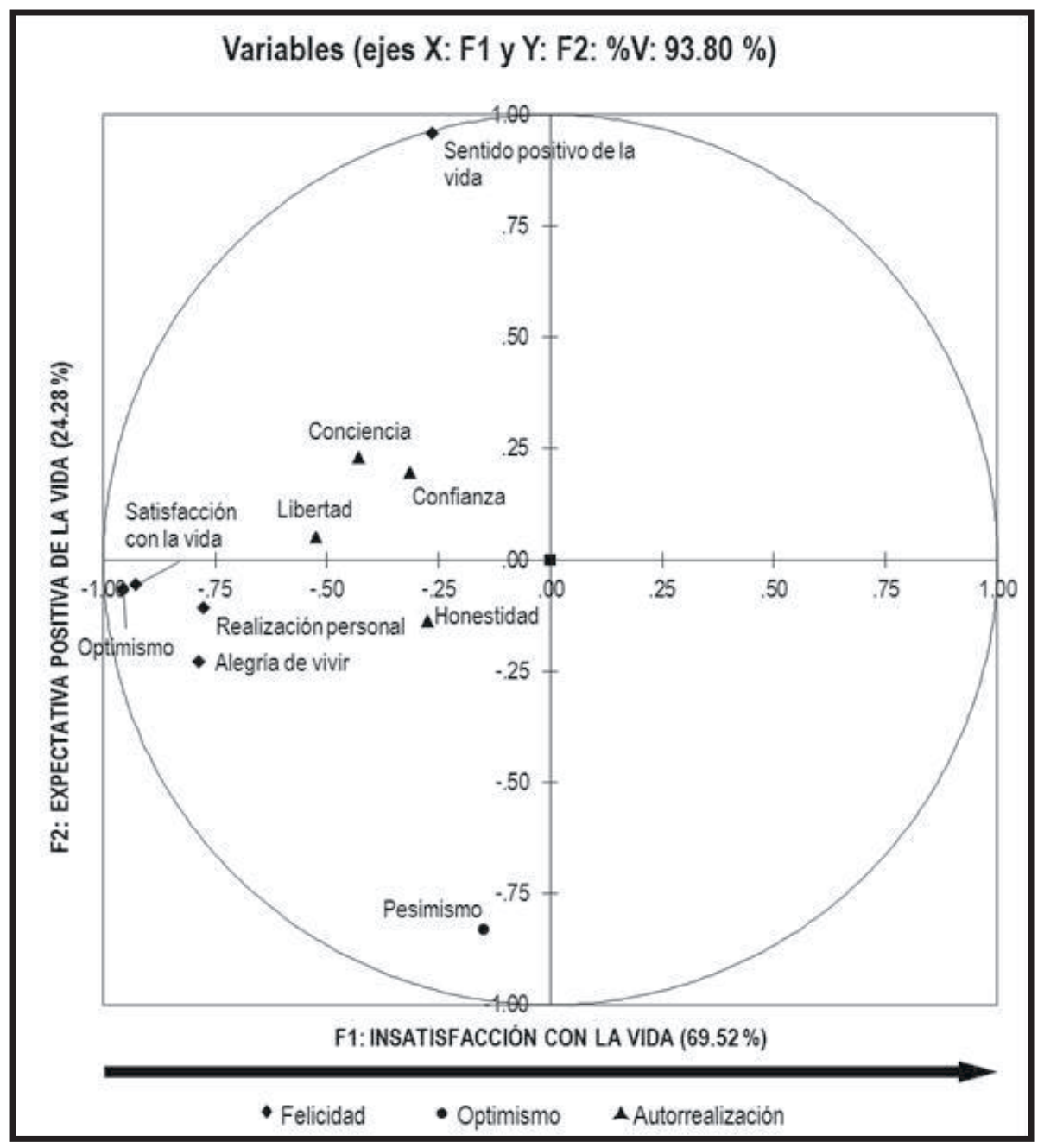




\section{REFERENCIAS BIBLIOGRÁFICAS}

Alarcón, R. (2000). Variables psicológicas asociadas a la felicidad [Psychological variables associated with happiness]. Revista Persona, 3, 147157.

Alarcón, R. (2006). Desarrollo de una Escala Factorial para Medir la Felicidad [Development of a Factorial Scale to Measure Happiness]. Revista Interamericana de Psicología, 40(1), 99-106.

Arequipeño, F. \& Lastra, H. (2016). Optimismo como factor protector de la ansiedad en estudiantes de Psicología de una universidad privada de Tarapoto [Optimism as a protective factor of anxiety in Psychology students from a private university in Tarapoto]. Apuntes Psicológicos, 1 (1), 58-67.

Biswas-Diener, R. \& Diener, E. (2006). The subjective well-being of the homeless, and lessons for happiness. Social Indicators Research, 76 (2), 185-205. http://dx.doi.org/10.1007/s11205005-8671-9

Burga, I., Bolaños, L., Gaspar, M., \& Jara, J. (2014). Escala APICE de LENI [APICE Scale LENI]. Tarapoto:Unión.

Castaño, E. \& Garín, A. (2012). Incorporación de personas adultas a los estudios universitarios: Aprendizaje permanente para arquitectos técnicos [Incorporation of adults into college studies: Permanent learning for technical architects]. Formación Universitaria, 5(3), 17-26. http://dx. doi.org/10.4067/S0718-50062012000300003

Casullo, M. (2002). Evaluación del bienestar psicológico en Iberoamérica [Psychological wellbeing evaluation in Latin America]. Buenos Aires, Argentina: Paidós.

Cuadra, H. \& Florenzano, R. (2003). El bienestar subjetivo: Hacia una Psicología Positiva [Subjective well-being: Towards a Positive Psychology]. Revista de Psicología de la Universidad de Chile, 12(1), 83-96.
Cruz, M., Chaves, M., Barcellos R., Almeida L., de Oliveira Secco, I. \& Jorge L. (2010). Exceso de trabajo y agravios mentales a los trabajadores de la salud [Overwork and mental health problems in health workers]. Revista Cubana de Enfermería, 26(1), 52-64.

Chang, E.C. \& Asakawa, K. (2003). Cultural variations on optimistic and pessimistic bias for self versus a sibling: Is there evidence for self-enhancement in the west and for self-criticism in the east when the referent group is specified? Journal of Personality and Social Psychology, 84, 569-581. http://dx.doi.org/10.1037/0022-3 514.84.3.569

Chitgian-Urzúa, V., Urzúa, A. \& Vera-Villarroel, P. (2013). Análisis preliminar de las escalas de bienestar psicológico en población chilena [Preliminary analysis of psychological well-being scales in Chilean population]. Revista Argentina de Clínica Psicológica, 32(1), 5-14.

Davidson, K.W., Mostofsky, E. \& Whang, W. (2010). Don't worry, be happy: Positive affect and reduced 10-year incident coronary heart disease: The Canadian Nova Scotia Health Survey. European Heart Journal, 31(9), 1065 1135. http://dx.doi.org/10.1093/eurheartj/eh p6 03

Díaz, D., Rodríguez, R., Blanco, A., Moreno, B., Gallardo, I., Valle, C. \& Van Dierendonck, D. (2006). Adaptación española de las Escalas de Bienestar Psicológico de Ryff [Spanish Adaptation of Ryff's Psychological Well-Being Scales]. Psicothema, 18, 572-577.

González, J. \& Restrepo, G. (2009). Prevalencia de felicidad en ciclos vitales y relación con redes de apoyo en población colombiana [Prevalence of happiness in life cycles and regarding support networks in Colombian population]. Revista de Salud Pública, 12(2), 228-238.

Grimaldo, P. (2004). Niveles de optimismo en un grupo de estudiantes de una universidad parti- 
cular de la Ciudad de Lima [Optimism levels in a group of students from a specific university of the city of Lima]. Liberabit, 1(10), 96-106.

Gustems, J. \& Sánchez, L. (2015). Aportaciones de la Psicología Positiva aplicadas a la formación del profesorado [Positive Psychology contributions applied to teachers' education]. Estudios sobre Educación, 29, 9-28. http://dx.doi.org/10. 15581/004.29.9-28

Hartog, J. \& Oosterbeek, H. (1996). Salud, riqueza y felicidad: ¿Por qué continuar con una educación superior? [Health, wealth and happiness: Why continue studying higher education?]. Trabajo presentado en el Congreso de la Asociación Económica Europea. Estambul.

Lyubomirsky, S. (2008). La ciencia de la felicidad [Happiness science]. Barcelona: Uranos.

Marrero, R.J., Carballeira, M. \& González, J.A. (2014). Relación entre bienestar subjetivo, optimismo y variables sociodemográficas en estudiantes universitarios de la Universidad de San Luis Potosí en México [Relationship between subjective well-being, optimism and sociodemographic variables in students of the University of San Luis Potosí in Mexico]. Universitär Psychologien, 13(3), 1083-1098. http://dx. doi. org/10.11144/Javeriana.UPSY13-3.rbso

Martínez, M. (2006). El estudio científico de las fortalezas trascendentales desde la Psicología Positiva [Scientific study of important strengthens from the Positive Psychology perspective]. Clínica y Salud, 17, 245-258.

Mestre, M.V., Samper, P., Frías, M.D. \& Tur, A.M. (2009). Are women more empathetic than men? A longitudinal study in adolescence. The Spanish Journal of Psychology, 12, 63-83.

Moyano, D.E., Flores, M.E. \& Soromaa, H. (2011). Fiabilidad y validez de constructo del Test Munsh para medir felicidad, en población de adultos mayores chilenos [Reliability and construct validity of Munsh Test to measure hap- piness in Chilean senior citizens]. Universitas Psychologica, 10, 567-580.

Muñoz Valdés, Y., Poblete Tolosa, Y. \& Jiménez Figueroa, A. (2012). Calidad de vida familiar y bienestar subjetivo en jóvenes con discapacidad intelectual de un establecimiento con educación especial y laboral de la Ciudad de Talca [Quality of family life and subjective well-being in young people with intellectual disability from a special education school and work of the city of Talca]. Interdisciplinaria, 29(2), 271-286. http: //dx.doi.org/10.16888/interd.2012.29.2.1

Mustaca, A., Kamenetzky, G. \& Vera-Villarroel, P. (2010). Relaciones entre variables positivas y negativas en una muestra de estudiantes argentinos [Relationships between negative and positive variables in an Argentinean students sample]. Revista Argentina de Clínica Psicológica, 19, 227-335.

Naranjo, M. (2007). Autoestima: Un factor relevante en la vida de la persona y tema esencial del proceso educativo [Self-esteem: A relevant factor in the life of a person and essential matter of the education process]. Actualidades Investigativas en Educación, 7(3), 1-27. http://dx.doi. org/10.15517/aie.v7i3.9296

Oros, L. \& Fontana, A. (2015). Niños socialmente hábiles: ¿Cuánto influyen la empatía y las emociones positivas? [Socially skillful children: How much influence do empathy and positive emotions have?]. Interdisciplinaria, 32(1), 109125. http://dx.doi.org/10.16888/interd.2015.32. 1.6

Otero López, J.M., Luengo, A., Romero, E., Gómez, J.A. \& Castro, C. (1998). Psicología de la personalidad. Manual de prácticas [Personality Psychology. Practices manual]. Barcelona: Ariel Practicum.

Park, N. \& Peterson, C. (2009). Achieving and sustaining a good life. Perspectives on Psychological Science, 4, 422-428. 
Pavez, P., Mena, L. \& Vera-Villarroel, P. (2012). El rol de la felicidad y el optimismo como factor protector de la ansiedad [The role of happiness and optimism as a protective factor against anxiety]. Universitas Psychologica, 11 (2), 369-380.

Peterson, C. (2000). The future of optimism. American Psychologist, 55, 44-55. http://dx.doi.org/ 10.1037//0003-066X.55.1.44

Remor, E., Amorós, M. \& Carboles, J. (2006). El optimismo y la experiencia de ira en relación con el malestar físico [Optimism and experience of anger in relation with physical discomfort]. Anales de Psicología, 22, 37-44.

Ryff, C. (1989). Happiness is everything: or is it? Explorations of meaning of psychological wellbeing. Journal of Personality and Social Psychology, 57, 1069-1081. http://dx.doi.org/10.1 037/0022-3514.57.6.1069

Ryff, C., Keyes, C. \& Shmotkin, D. (2002). Optimizing well-being: The empirical encounter of two traditions. Journal of Personality and Social Psychology, 82, 1007-1022. http://dx.doi. org/10.1037//0022-3514.82.6.1007

Salgado, C. (2009). Felicidad, resiliencia y optimismo en estudiantes de colegios nacionales de la ciudad de Lima [Happiness, resilience and optimism in public school students of the city of Lima]. Liberabit, 15(2), 133-141.

Seligman, M.E.P. (2002). Authentic happiness: Using the new positive psychology to realice your potential for lasting fulfillment. Nueva York: Free Press.

Seligman, M. (2003). La auténtica felicidad [The authentic happiness]. Barcelona: Ediciones Vergara.

Seligman, M. E. P. \& Csikszentmihalyi, M. (2000). Positive psychology: An introduction. American Psychologist, 55, 5-14. http://dx.doi.org/ 10.1037//0003-066X.55.1.5
Torres, M., Moyano, E. \& Páez, D. (2014). Comportamiento juvenil universitario en busca de la felicidad: Su caracterización y su eficacia [Behavior of college students in search of happiness: Its characterization and efficacy]. Universitas Psychologica, 13(4), 15-24. http://dx. doi.org/10.11144/Javeriana.UPSY13-4.cjub

Vázquez, C., Hervás, G. \& Ho, S.M.Y. (2006). Intervenciones clínicas basadas en la psicología positiva: Fundamentos y aplicaciones [Clinical Interventions based on Positive Psychology: Fundamentals and applications]. Psicología Conductual, 14(3), 401-432.

Vecina, M. (2006). Emociones positivas [Positive emotions]. Papeles del Psicólogo, 27, 9-17.

Vera-Villarroel, P., Celis -Atenas, K., Pavez, P., Lillo, S., Bello, F., Díaz, N. \& López, W. (2012). Money, age and happiness: Association of well being with sociodemographic variables. Revista Latinoamericana de Psicología, 44(2), 155-163. Vera-Villarroel, P., Córdova-Rubio, N. \& CelisAtenas, K. (2009). Evaluación del optimismo: Un análisis preliminar del Life Orientation Test versión revisada (LOT-R) en población chilena [Optimism evaluation: A preliminary analysis of the Reviewed Version of Life Orientation Test (LOT-R) in Chilean population]. Universitas Psychologica, 8, 61-68.

Vera-Villarroel, P., Pavez, P. \& Mena, L. (2012). El rol de la felicidad y el optimismo como factor protector de la ansiedad [The role of happiness and optimism as a protective factor against anxiety]. Universitas Psychologica, 11(2), 369380.

Vera-Villarroel, P., Pávez, P. \& Silva, J. (2012). El rol predisponente del optimismo: Hacia un modelo etiológico del bienestar [The predisposing role of optimism: Towards an etiological model of well-being]. Terapia Psicológica, 30(2), 7784. http://dx.doi.org/10.4067/S0718-48082012 000200008 
Wood, A. \& Joseph, S. (2010). The absence of positive psychological (eudemonic) well-being as risk factor for depression: A ten years cohorte stydy. Journal of Affective Disordes, 122(3), 213-217. http://dx.doi.org/10.1016/j.jad.2009. 06.032

Zúñiga, J. (2010). Incidencia del ejercicio físico / deportivo sobre el bienestar psicológico [Inci- dence of physical / sports exercise on psychological well-being]. Tesis de Licenciatura no publicada. Universidad del Aconcagua, Argentina. Recuperado el 28 de marzo de 2016 de http://bibliotecadigital.uda.edu.ar/objetos_digi tales/51/tesis- 995- incidencia.pdf

Universidad Peruana Unión (Tarapoto) Universidad San Ignacio de Loyola (Lima)

Perú

Fecha de recepción: 4 de mayo de 2016 Fecha de aceptación: 28 de septiembre de 2016 